\title{
Identifying Non-transiting Terrestrial Planets with Transit Timing Data
}

\author{
Dimitri Veras and Eric B. Ford \\ Department of Astronomy, University of Florida, \\ 211 Bryant Space Science Center, P.O. Box 112055, Gainesville, FL 32611-2055 \\ email: veras@astro.ufl.edu, eford@astro.ufl.edu
}

\begin{abstract}
Both ground and space-based transit observatories are poised to significantly increase the number of known transiting planets and the number of precisely measured transit times. A planet's transit times represent a clock that can be used to infer the presence of additional planets. Although modeling the transit time variations (TTVs) of a known system is simple, interpreting those variations in terms of the mass and orbital elements of a perturbing planet is much more challenging. Because mutual planetary perturbations are often the dominant source of TTVs, the observable signal can be extremely complex. In these proceedings, we present early results based on a simplistic analysis of the root-mean-square TTV deviation amplitude. We are preparing a more thorough analysis based on a computationally efficient surrogate Bayesian model, which may be combined with analytic approximations and n-body integrations in order to establish the sensitivity of TTV observations to terrestrial-like planets as a function of the system architecture. Besides aiding the interpretation of future transit timing observations, we hope our results can help maximize the productivity of transit timing follow-up campaigns by guiding survey design decisions such as the choice of targets, required precision, and desired number/time span of TTV observations.
\end{abstract}

\section{Introduction}

The mounting number of transiting exoplanet detections suggest that multi-planet systems containing at least one known transiting planet will become commonplace. Observed transit timing variations can indicate the presence of non-transiting planets in such systems. Agol et al. (2005) and Holman \& Murray (2005) consider possibilities for detecting terrestrial-mass non-transiting planets, and Steffen \& Agol (2005) and Agol \& Steffen (2007) focus these considerations on two specific systems, TrES-1 and HD 209458. TTVs may detect the long-term secular advance of a planet's pericenter, which could imply either the presence of an additional, perturbing planet, and/or provide an estimate of the parent star's oblateness (Heyl \& Gladman, 2007). TTVs may also be detectably affected by general relativity (Miralda-Escudé, 2002, Pál \& Kocsis, 2008) and may help discover extrasolar moons (Doyle \& Deeg, 2004, Simon et al., 2007) and/or Trojan planets (Ford \& Holman, 2007). Although new theoretical treatments incorporating added physics and better characterizing resonant configurations (e.g. Mardling, 2008, Pál \& Kocsis, 2008) are helping this nascent field gain solid footing, many mysteries remain.

In particular, in nearly all cases, if or how a given TTV curve combined with a known transiting planet can pinpoint the mass and orbital parameters of an external perturber remains a mystery. This study outlines a method which makes progress towards solving this problem, and preliminary results are presented. 


\section{Methods}

We consider a Jupiter-mass planet which transits its parent Solar-mass star on a circular orbit $\left(e_{2}=0\right)$ at $a_{2}=0.05 \mathrm{AU}$, and an external Earth-mass planet with semimajor axis, $a_{1}$, and eccentricity, $e_{1}$. After fixing initial values for $a_{1}, e_{1}$, and the planets' orbital angles, we integrate the system over 10 years and note the instances of transits. From that information, we construct a transit timing variation curve. The transit points along this curve are not exactly uniformly spaced, and the deviations from strict periodicity tend to form periodic patterns. In principle, we may select any combination of transits to mimic observed data. Here, we select the TTV curves produced by the first 50 instances of transits for the below results.

TTV profiles may change - both in amplitude and frequency - by several orders of magnitude when the initial values of the mean anomalies and longitudes of pericenter are varied. This property of TTV profiles complicates the process of identifying the orbit of the external perturber. A detailed study of the correlation of orbital angles with TTV profiles is warranted for application of our method to a real system. Here, we introduce our methodology by simulating a representative sample of initial orbital angles for each of the fixed values of semimajor axes and eccentricity. Figure 1 displays the median root-mean-square (RMS) TTV deviation amplitude (in seconds) of 10 systems initialized with random mean anomalies and longitude of pericenters of both planets, for different $a_{1}$ and $e_{1}$ values. We sampled 400 logarithmically-spaced values of the semimajor axis and uniformly-spaced values of $e_{1}$ for each semimajor axis ratio. We only sampled systems guaranteed to be stable according to the Hill Stability Limit (Gladman, 1993). Labeled vertical lines on the figure mark locations of low-order mean motion commensurabilities between both planets. Note the staggered scale of the contours, which were chosen to enhance the most dynamically relevant portions of phase space.

The contour plot demonstrates that several different dynamical regimes may exhibit the same TTV RMS amplitude. Increasing the eccentricity of the outer perturber will increase the TTV signal in most cases, except sometimes at semimajor axis ratios which correspond to near-exact resonance. In regions away from resonance, the TTV RMS amplitude typically varies from a few seconds to a few $10^{3}$ seconds depending on the value of $e_{1}$. We expect stable systems which may lie above the stability curve in Fig. 1 away from resonance to also exhibit TTV RMS amplitudes that exceed $10^{3}$ seconds.

Resonances play a key role in sculpting the contours of Fig. 1. Gravitational scattering studies of giant planets have demonstrated that resonances of up to 3rd-order may help shape the architecture of a system (Veras \& Armitage, 2004). TTV signals are significantly more sensitive to low-order resonances, as commensurabilities of at least order 8 correspond to signatures in Fig. 1. The TTV RMS amplitude may be greater just off-resonance rather than at exact resonance. A resonance's radial signature - a type of "libration width" - may be estimated by formulas given in Murray \& Dermott (1999) and Veras \& Armitage (2004). Additional simulations indicate that the TTV RMS amplitudes scale linearly with the mass of the external perturber, and hence determine how pronounced each resonant signature is in Fig. 1.

\section{Conclusion and Implications}

Our study contributes a first step towards identifying a second planet in an exosystem with one known transiting planet. Through an extensive sampling of the eccentricitysemimajor axis phase space of an external terrestrial-mass planet coplanar with the transiting planet, we can determine the median amplitude and frequency of TTV signals 


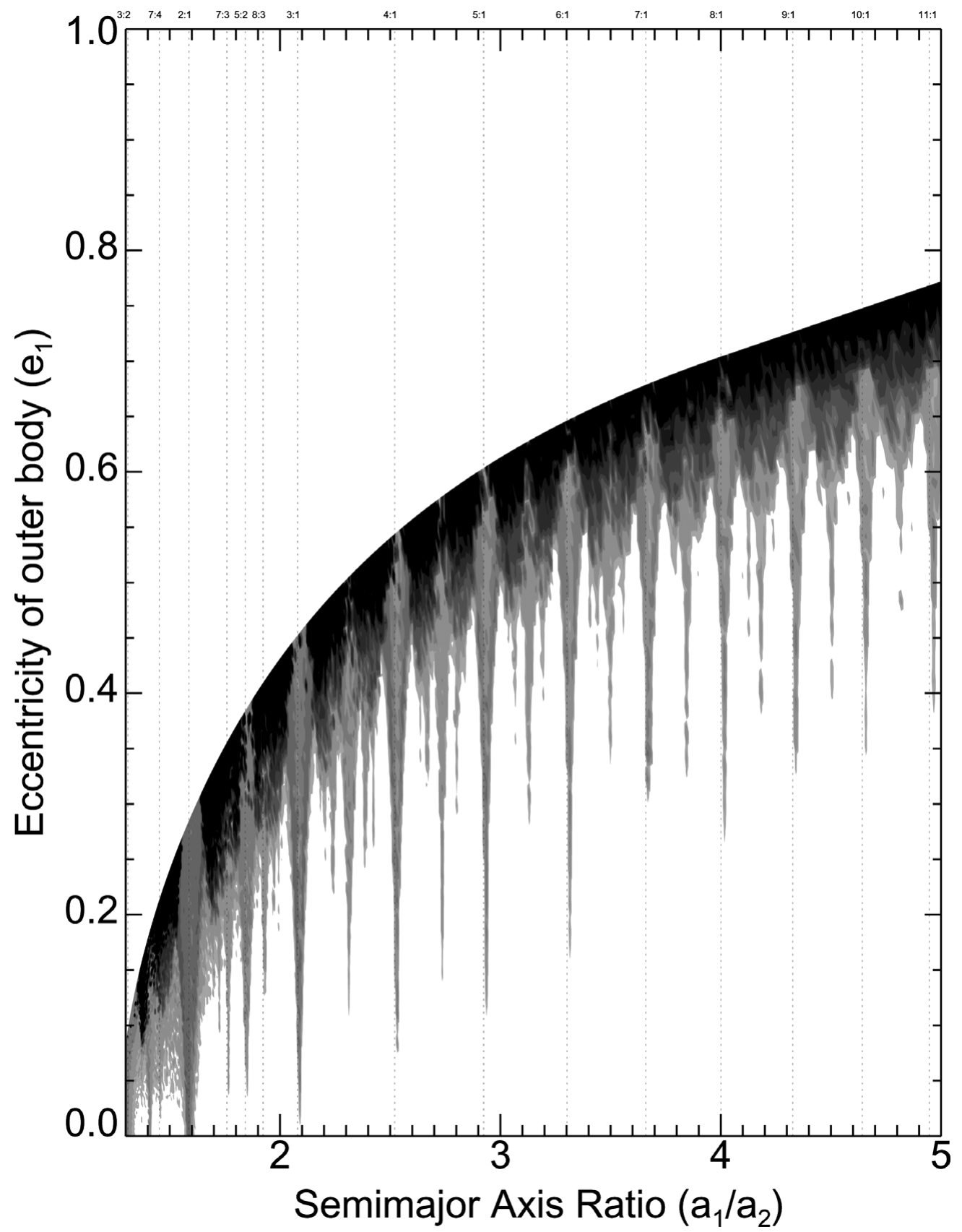

Figure 1. The RMS deviations of TTV signals is sensitive to the semi-major axis and eccentricity of the perturbing planet. The figure above shows the deviations induced by an outer planet of 1 Earth mass whose semi-major axis and eccentricity correspond to the $\mathrm{x}$ - and $\mathrm{y}$-axes. The gradation indicates the median RMS TTV deviation amplitude (in seconds) of 10 systems with the specified orbital parameters. The lightest to darkest shades correspond to, in seconds, [10-20], [20-50], [50-100], [100-200], [200-500], [500-1000], [1000-2000], [2000-3000], [3000-4000], [4000-5000] and [5000-6000]. Simulations assume 50 consecutive transits are timed with 10-second precision. The upper envelope defines the Hill stability limit. TTV signals are easily detectable at or near several mean motion resonances (indicated by vertical lines). 
produced by each possible geometry. We find that the TTV signals may be highly sensitive to the initial orbital positions of both planets, and vary over several orders of magnitude. The degeneracy produced by the initial orbital angles may be partially or fully broken by utilizing the frequencies of the TTV signal.

Because the TTV profile is a sensitive function of the number and frequency of transits observed, we hope to follow up this initial study by modeling a greater variety of realistic data sets. Preliminary results indicate that Fig. 1 remains largely unchanged for data sets comprised of 30 or 40 roughly equally spaced transits. For certain orbital configurations, as few as 10 transit detections may be able to constrain TTV profiles well enough to place useful constraints on the orbital parameters of a possible external perturber.

This work was supported by NSF grant AST-0707203 and the University of Florida.

\section{References}

Agol, E. \& Steffen, J. H. 2007, MNRAS, 374, 941

Agol, E., Steffen, J., Sari, R., \& Clarkson, W. 2005, MNRAS, 359, 567

Doyle, L. R. \& Deeg, H.-J. 2004, Bioastronomy 2002: Life Among the Stars, 213, 80

Ford, E. B. \& Holman, M. J. 2007, Ap. Lett., 664, L51

Gladman, B. 1993, Icarus, 106, 247

Heyl, J. S. \& Gladman, B. J. 2007, MNRAS, 377, 1511

Holman, M. J. \& Murray, N. W. 2005, Science, 307, 1288

Mardling, R. A. 2008, Astro-ph arXiv:0805.1928

Miralda-Escudé, J. 2002, ApJ, 564, 1019

Murray, C. D. \& Dermott, S. F. 1999, Solar System Dynamics

Pál, A. \& Kocsis, B. 2008, Astro-ph, arXiv:0806.0629

Simon, A., Szatmáry, K., \& Szabó, G. M. 2007, A $\& A$, 470, 727

Steffen, J. H. \& Agol, E. 2005, MNRAS, 364, L96

Veras, D. \& Armitage, P. J. 2004, Icarus, 172, 349 